\title{
Sull'informazione associata alle esperienze incomplete (*).
}

\author{
B. Forte $\theta$ N. Pintacuda (Pavia).
}

\begin{abstract}
Sunto. - Nell'ambito della teoria dell' informazione senza probabilità, si propone una definizione assiomatica dell' informazione associata ad esperienze incomplete.

Si discutono in particolare lo proprietà del guadagno di informazione e dell' infor" mazione condizionale, ginngendo a caratterizade con un sistema di equazioni funzionali la classe delle misure di informazione che soddisfano agli assiomi adottati; come caso particolare si ottiene la classica misura di Shannon-Rényi.
\end{abstract}

\section{Introduzione.}

Il problema della misura della quantità di informazione è stato classicamente affrontato da due punti di vista distinti.

Dato un insieme $\Omega$ di eventi elementari $\omega$ e una famiglia of di parti (eventi) di $\Omega$, si considera, secondo il primo punto di vista, l'informazione che si ha dal manifestarsi in un evento $A \in \mathscr{F}$. In base al secondo punto di vista, data una famiglia $\mathcal{G}$ di esperienze, ciò data una famiglia di partizioni II di $\Omega$ in un numero finito qualsivoglia di eventi $A_{1}, A_{2}, \ldots, A_{n}\left(A_{i} \in \mathscr{F}\right)$, si considera l'informazione $H$ (II) che si ha da tali esperienze, ovvero dal manifestarsi di un evento qualsiasi, peraltro non precisato, degli eventi $A_{i}$ che la formano.

Particolare significato acquistano questi due punti di vista nella teoria dei questionari [1], per la quale il singolo evento $A$ è l'insieme delle risposte favorevoli ad una data domanda, mentre la partizione $I$ corrisponde ad un insieme di domande mutuamente esolnsive.

In alcune recenti note [2], J. KaMPÉ DE FÉRIET e B. ForTw hanno sug. gerito di generalizzare la definizione classica di Wiener-Shannon per la misura $J(A)$ della quantità di informazione associata ad un evento; nello stesso ambito di generalita, con il presente lavoro - i cui principali risultati sono stati annunciati tramite una nota ai Comptes Rendus - intendiamo suggerire una definizione della misura della quantità di informazione $H(\Pi)$ associata ad una esperienza; adotteremo tra l'altro il punto di vista più generale assunto da A. Rínyi [3], e considereremo esperienze incomplete, vale a dire definiremo $h(\Pi)$ quando $\Pi$ è una partizione di un evento $A \subset \Omega(A \in \mathfrak{F})$.

$\left({ }^{*}\right)$ Lavoro eseguito per la realizzazione del programma del gruppo di ricerca $n .7$ del C. N. R.. 
Questa definizione contiene orviamente il caso in eui $A$ coincide con $\Omega$, nel qual caso la partizione II sarà chinmata esperienza completa.

L'accennato spirito di generalità consiste nella circostanza che la definizione proposta è del tutto indipendente dalla esistenza di una misura di probabilità per gli eventi $A \in \mathscr{F}$.

Naturalmente, verrà dimostrato che la classica misura dell'informazione di Shannon-Rényi in funzione delle probabilità dei singoli eventi rientra co. me caso particolare.

Verrà altresi data una condizione sufficiente perchè la quantită di informazione associata ad un'esperienza incompleta sia quella di Shannon-Rényi.

\section{1. - Le esperienze.}

Supponiamo data una famiglia $\mathscr{F}$ di parti $A$ di un insieme fondamentale $\Omega$, comprendente $\Omega \in Q$. Sia $\mathcal{E}$ una famiglia non vuota di insiemi finiti di elementi disgiunti di $\mathfrak{F}$.

Gli elementi di $\mathcal{E}$ saranno chiamati esperienze; un'esperienza II $\in \mathcal{E}$ per la quale sia $\underset{A \in U}{\bigcup} A=\Omega$ sard̀ detta completa.

Introduciamo per gli elementi di \& le due seguenti relazioni d'ordine:

a) $\quad \Pi_{1}<\Pi_{2}, \quad$ se $\underset{A^{\prime} \in \Pi_{1}}{\cup} A^{\prime}=\underset{A^{\prime \prime} \in \Pi_{2}}{\bigcup} A^{\prime \prime} \quad$ e inoltre $\forall A^{\prime} \in \Pi_{2} \exists A^{\prime}: A^{\prime \prime} \subset A^{\prime} \in \Pi_{1}$

e leggiamo $\Pi_{1}<\Pi_{2}$ dicendo che l'esperienza $\Pi_{1}$ è meno fine dell'esperienza $\Pi_{2}$. Questa relazione d'ordine, come si può facilmente verificare, è riflessiva.

$$
\Pi_{1} \subset \Pi_{2} \quad \text { se } \quad \forall A^{\prime} \in \Pi_{1} \Rightarrow A^{\prime} \in \Pi_{2}
$$

che d'altra parte è la relazione di inclusione nel senso della teoria degli insiemi.

Introduciamo inoltre per gli elementi di $\mathcal{G}$ le operazioni seguenti:

$$
\Pi_{1} \cdot \Pi_{2}=\left\{A: A=A^{\prime} \cap A^{\prime \prime}, \quad A^{\prime} \in \Pi_{1}, \quad A^{\prime \prime} \in \Pi_{2}\right\}
$$

2) se $\left(\bigcup_{A^{\prime} \in \Pi_{2}}^{\cup} A^{\prime}\right) \cap\left({\underset{A}{\prime \prime} \in \Pi_{2}}_{\cap^{\prime \prime}} A^{\prime}=0\right.$, porremo $\Pi_{1} \cup \Pi_{2}=\left\{A: A \in \Pi_{1} \quad\right.$ oppure $\left.A \in \Pi_{2}\right\}$

operazione che d'altra parte è l'unione di $\Pi_{1}$ e $\Pi_{2}$ nel senso della teoria degli insiemi.

Si verifica immediatamente ohe il prodotto $\Pi_{1} \cdot \Pi_{2}$ è associativo, commutativo, distributivo rispetto a $U$, e monotono rispetto all'ordine $<$, vale a dire

$$
\Pi_{1}<\Pi_{1}^{\prime}, \quad \Pi_{2}<\Pi_{2}^{\prime} \quad=>\quad \Pi_{1} \cdot \Pi_{2}<\Pi_{1}^{\prime} \cdot \Pi_{2}^{\prime} .
$$


Assegniamo pure a priori un sottoinsieme $\mathscr{K}$ di $\mathcal{G}$, che sarà detto l'insie. me delle esperienze indipendenti, con la proprietà che le algebre di Boole generate $\mathrm{da}$ due qualsiasi elementi di $\mathfrak{d}$ siano indipendenti in senso algebrico [4].

Sia inoltre $N(I)$ il numero delle parti disgiunte che formano l'esperienza II; $N(\Pi)$ è per ipotesi sempre finito.

Detto $\mathcal{E}_{n}$ il sottoinsieme di $\mathcal{E}$ formato dalle esperienze II per le quali $N(\Pi)=n$, si ha

$$
\mathcal{E}=\bigcup_{n \geq 0} \mathcal{E}_{n}
$$

D'ora in poi supporremo che, per ogni $n, \mathcal{E}_{n}$ sia non vuoto; supporremo infine verificata da $\mathcal{E}$ e da $\mathfrak{d}$ le proprietà seguenti:

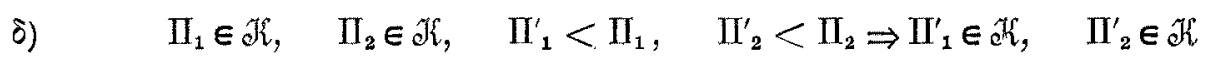

\section{2. - Misura dell'informazione.}

La misura della quantità di informazione fornita da un'esperienza $\Pi I \in \mathcal{G}$ è un'applicazione $\mathscr{G} \stackrel{H}{\longrightarrow} \bar{R}^{+}$con le seguenti proprietà :

i) (monotonia):

$$
\Pi_{1} \in \mathcal{G}, \quad \Pi_{2} \in \mathcal{E}, \quad \Pi_{1}<\Pi_{2} \Rightarrow H\left(\Pi_{1}\right) \leq H\left(\Pi_{2}\right)
$$

ii) (additività sulle esperienze indipendenti):

$$
\Pi_{1} \in \mathfrak{H}, \quad \Pi_{2} \in \mathfrak{d}, \quad \Pi_{1} \cdot \Pi_{2} \in \mathcal{B} \Rightarrow H\left(\Pi_{1} \cdot \Pi_{2}\right)=H\left(\Pi_{1}\right)+H\left(\Pi_{2}\right)
$$

iii) (carattere locale dell'informazione):

$$
\mathrm{II}_{1}^{\prime} \cup \Pi_{2}^{\prime} \in \mathcal{G} \Rightarrow H\left(\Pi_{1}^{\prime} \cup \Pi_{2}^{\prime}\right)-H\left(\Pi_{1}^{\prime} \cup \Pi_{2}\right)=H\left(\Pi_{1} \cup \Pi_{2}^{\prime}\right)-H\left(\Pi_{1} \cup \Pi_{2}\right)
$$

\section{3. - Prime proprietà della misura dell’informazione.}

Gli assiomi i) - iii) implicano le proprietà seguenti:

$$
\text { se } \quad \Pi \in \mathcal{E} \quad \text { e } \quad \Pi^{*}=\Pi \cup\{\boldsymbol{Q}\} \in \mathcal{E}, \quad \text { allora } \quad H\left(\Pi^{*}\right)=H(\Pi)
$$

per riconoscerlo, basta osservare che si ha simultaneamente $\Pi<\Pi^{*}, \Pi^{*}<\Pi$, donde per l'assioma i) è $H(\Pi) \leq H\left(\Pi^{*}\right), H\left(\Pi^{*}\right) \leq H(\Pi)$; da ciò l'asserto. 
2) la restrizione a $\mathscr{E}_{n}$ dell'applicazione $\mathscr{E} \stackrel{I I}{\longrightarrow} \bar{R}^{+}$definisce una funzione reale $H_{n}\left(A_{1}, A_{2}, \ldots, A_{n}\right),\left(A_{1}, A_{2}, \ldots, A_{n}\right\} \in \mathcal{E}$, simmetrica rispetto alle $n$ variabili $A_{1}, A_{2}, \ldots, A_{n}$.

In effetti, un'esperienza è individuata dalle parti che la formano e non dall'ordine nel quale queste parti vengono prese in considerazione.

3)

$$
H_{n+2}\left(A_{1}, A_{2}, \ldots, A_{n}, Q\right)=H_{n}\left(A_{1}, A_{2}, \ldots, A_{n}\right)
$$

È un'immediata conseguenza della proprietà 1).

Per l'assioma"iii) è in particolare

$$
\begin{aligned}
& H_{n}\left(A_{1}, A_{2}, \ldots, A_{n-2}, A_{n-1}, A_{n}\right)-H_{n-1}\left(A_{1}, A_{2}, \ldots, A_{n-1} \cup A_{n}\right)= \\
& \quad=H_{3}\left(A_{1} \cup \ldots \cup A_{n-2}, A_{n-1}, A_{n}\right)-H_{2}\left(A_{1} \cup \ldots \cup A_{n-2}, A_{n-1} \cup A_{n}\right)
\end{aligned}
$$

Da quest'ultima proprietà si riconosce che la differenza

$$
H_{n}\left(A_{1}, A_{2}, \ldots, A_{n-2}, A_{n-1}, A_{n}\right)-H_{n-1}\left(A_{1}, A_{2}, \ldots, A_{n-1} \cup A_{n}\right)
$$

è una funzione $\Phi$ degli eventi $\bigcup_{i=1}^{n-2} A_{i}, A_{,-1}, A_{n}$.

Chiameremo questa differenza guadagno di informazione relativo al passaggio dalla partizione $\left\{A_{1}, A_{2}, \ldots, A_{n-2}, A_{n-1} \cup A_{n}\right\}$ alla partizione, più fine, $\left\{A_{1}, A_{2}, \ldots, A_{n}\right\}$.

\section{4. - Decomposizione dell'informazione.}

La proprietà 5) del precedente paragrafo conduce al seguente

Thorema 1. - Se H è una misura delle quantità di informazione fornite dalle esperienze di una data famiglia $\mathcal{E}$, per la sua restriaione $H_{n}$ a $\mathcal{E}_{n}$, vale la espressione seguente:

$$
H_{n}\left(A_{1}, \ldots, A_{n}\right)=H_{1}\left(\bigcup_{k=1}^{n} A_{k}\right)+\sum_{i=0}^{n-2} \Phi\left(\bigcup_{k=0}^{i} A_{k}, A_{i+1}, \bigcup_{k=i+2}^{n} A_{k}\right)
$$

dove si è posto $A_{0}=Q$.

Dimostrazione. - Basta applicare iterativamente la proprietà 5).

In virtù della 4.1, l'informazione $H(I)$ associata ad una esperienza (incompleta) $\Pi \equiv \equiv\left\{A_{1}, \ldots, A_{n}\right\}$ risulta decomposta nella somma di una funzione di evento

$$
h\left(\bigcup_{k=1}^{n} A_{k}\right)=H_{1}\left(\bigcup_{k=1}^{n} A_{k}\right)
$$


e di una particolare funzione

$$
Z(I)=\sum_{i=0}^{n-2} \Phi\left(\bigcup_{k=0}^{i} A_{k}, A_{i+1}, \bigcup_{h=i+2}^{i} A_{h}\right)
$$

L'applicazione $\mathcal{E} \stackrel{H}{\rightarrow} \bar{R}^{+}$risulta così univocamente determinata dalla sua restrizione ad $\mathfrak{G}_{1}$, e dal guadagno di informazione $\Phi$. Ne segue che si possono tradurre le proprieta i) - iii) che definiscono detta applicazione $H$ in condizioni sulla funzione di evento $h(A)$ e sul guadagno di informazione $\Phi$.

E anche utile osservare che, posto $Z(\{A\} \cup\{Q\})=Z(; A\})$, e quindi $Z(\lfloor A)=0$, la applicazione $\mathscr{G} \stackrel{Z}{\longrightarrow} R+$ soddisfa, come si può facilmente verificare, gli as. siomi i) - iii) ed è quindi anch'essa una misura della quantità di informazione associata alle esperienze di $\mathcal{E}$.

\section{5. - Proprietà della funzione $h$ e informazione condizionale.}

Tenuto conto della definizione della funzione di evento $h(A)$ per la quale $h(A)=H_{1}(A)$, si ha immediatamente:

a) $h(A) \in \bar{R}^{+}$

b) $h(A \cap B)=H_{1}(A \cap B)=H_{1}(A)+H_{1}(B)=h(A)+h(B)$ se $\{A\} \in \mathfrak{d},\{B \in \mathfrak{K} \quad$ (additività sugli eventi indipendenti).

Se si suppone inoltre - proprietà tuttavia non implicata dagli assiomi i) - iii) - che $h(A)$ sia una funzione di evento non decrescente, cioè se si suppone ulteriormente che

$$
\text { e) } A \subset B \Rightarrow h(A) \geq h(B)
$$

le proprietă a) b) c) di $h$ coincidono con quelle che definiscono l'informazione di un evento secondo J. Kampé de Fériet e B. Forte.

In questo caso particolare, la differenza

$$
H_{n}\left(A_{1}, \ldots, A_{n}\right)-H_{1}\left(\bigcup_{k=1}^{n} A_{k}\right)=Z(\Pi)
$$

che è il guadagno di informazione per il passaggio dall'informazione fornita dall'esperienza $\left\{\bigcup_{k=L}^{n} A_{k}\right\}$ a quella fornita dalla esperienza più fine $\left\{A_{1}, \ldots, A_{n}\right\}$ si può altresì interpretare come l'informazione associata all'esperienza I, quando si sappia che si è manifestato l'evento $\bigcup_{k=1}^{n} A_{k}$. 
Pertanto l'applicazione $\mathcal{E} \stackrel{Z}{\longrightarrow} \bar{R}^{+} \dot{\ominus}$ l'informazione condizionale dell'esperienza $\&$ dato l'evento $\left\{\bigcup_{k=1}^{n} A_{k}\right.$.

Per questo motivo, sulla scorta dell'osservazione finale del paragrafo precedente, anche nel caso in cui $h(A)$ non soddisfi la proprietà $c$ ), chiameremo ancora la quantità $H_{n}\left(A_{1}, \ldots, A_{n}\right)-h\left(\cup A_{k}\right)$ informazione condizionale di $\Pi \equiv\left\{A_{1}, \ldots, A_{n}\right\}$ dato l'evento $\bigcup_{k=1}^{\bigcup} A_{k}$.

\section{6. - Proprietà del guadagno di informazione $\Phi$.}

Il guadagno di informazione $\Phi$ è come si è visto una applicazione in $\bar{R}^{+}$delle terne di eventi disgiunti $A, B, C$ tali che $\{A, B, C\} \in \mathcal{G}$.

L'assioma i) del paragrafo 2 e la proprietà 4) del paragrafo 3 implicano:

I.

$$
\Phi(A ; B, C) \geq 0
$$

Per la proprietà 3) del paragrafo 3 si ha poi

II.

$$
\Phi(A ; Q, C)=0 .
$$

Se si applica ora la proprieta 4 ) del paragrafo 3 alla esperienza $\Pi \equiv$ $\equiv\{A, B, C, D\}$ e si ricorda la proprietà di simmetria della funzione $H_{n}$ (proprietà 2 del paragrafo 3 ), si ha

$$
\begin{aligned}
& H_{4}(A, B, C, D)-H_{3}(A \cup B, C, D)=\Phi(C \cup D ; A, B) \\
& H_{3}(A \cup B, C, D)-H_{2}(A \cup B \cup C, D)=\Phi(D ; A \cup B, C)
\end{aligned}
$$

e sommando membro a membro

$$
H_{4}(A, B, C, D)-H_{2}(A \cup B \cup C, D)=\Phi(C \cup D ; A, B)+\Phi(D ; A \cup B, C) .
$$

D'altra parte si ha pure

$$
\begin{gathered}
H_{4}(A, C, B, D)-H_{3}(A \cup C, B, D)=\Phi(B \cup D, A, C) \\
H_{3}(A \cup C, B, D)-H_{2}(A \cup C \cup B, D)=\Phi(D ; A \cup C, B)
\end{gathered}
$$

da cui

(6.6) $H_{4}(A, C, B, D)-H_{2}(A \cup C \cup B, D)=\Phi(B \cup D ; A, C)+\Phi(D ; A \cup C, B)$ 
Confrontando (6.3) con (6.6), si ha infine:

III. $\Phi(C \cup D ; A, B)+\Phi(D ; A \cup B, C)=\Phi(B \cup D, A, C)+\Phi(D ; A \cup C, B)$

Si noti che dalle proprietà II. e III. si deduce in particolare, ponendo $A=Q$,

$$
\Phi(D ; B, C)=\Phi(D ; C, B)
$$

vale a dire che $\Phi$ è una funzione simmetrica dei suoi ultimi due argomenti.

L'additività dell'informazione sul prodotto di esperienze indipendenti porta ad una ulteriore condizione sulla funzione $\Phi$.

Siano $\Pi_{1} \equiv\left\{A_{1}, A_{2}, A_{3}{ }^{\prime}\right.$ e $\Pi_{2} \equiv\left\{B_{1}, B_{2}, B_{3}\right\}$ due esperienze indipendenti.

Se si impone alla funzione

$$
Z(\Pi)=\sum_{i=0}^{n-2} \Phi\left(\bigcup_{k=0}^{i} A_{k}, A_{i+1}, \bigcup_{n=i+2}^{n} A h\right)
$$

di soddisfare all'assioma ii) si ha in effetti

$$
Z\left(\Pi_{1} \Pi_{2}\right)=Z\left(\Pi_{1}\right)+Z\left(\Pi_{2}\right)
$$

D'altra parte per la proprietà 4) del paragrafo 3 (conseguenza dell'assioma iii)) si ha

$$
\begin{aligned}
Z\left(\Pi_{1} \Pi_{2}\right)=Z\left(\left\{A_{1}, A_{2} \cup A_{3}^{\prime}\left\{B_{1}, B_{2},\right.\right.\right. & \left.\left.B_{3}\right\}\right)+\underset{k=1}{\stackrel{3}{Z}} \Phi\left(A_{\cap} B-\right. \\
& \left.-\left(A_{2} \cup A_{8}\right) \cap B_{k}, A_{2} \cap B_{k} ; A_{3} \cap B_{k}\right)
\end{aligned}
$$

D'altronde, tenuto conto delle ipotesi $\gamma$ ) e $\delta$ ) del paragrafo 1, si ha:

$$
Z\left(\left\{A_{1}, A_{2} \cup A_{3}\right\}\left\{B_{1} B_{2} B_{3}\right)=Z\left(\left\{A_{1}, A_{2} \cup A_{3}\right\}\right)+Z\left(\Pi_{2}\right)\right.
$$

confrontando le $(6.8),(6.9)$ e $(6.10)$, si ottiene

$$
\begin{aligned}
Z\left(\Pi_{1}\right)+Z\left(\Pi_{2}\right)=Z\left(\left\{A_{1}, A_{2} \cup A_{3}\right\}\right)+Z & \left.Z \Pi_{2}\right)+\sum_{k=1}^{3} \Phi(A \cap B- \\
& \left.-\left(A_{2} \cup A_{3}\right) \cap B_{k}, A_{2} \cap B_{k}, A_{3} \cap B_{k}\right)
\end{aligned}
$$

e infine

$$
\left.Z\left(\Pi_{1}\right)-Z Z\left(\left\{A_{1}, A_{2} \cup A_{3}\right\}\right)=\sum_{k=1}^{3} \Phi\left(A_{\cap} B-\left(A_{2} \cup A_{3}\right) \cap B_{k}\right) ; A_{2} \cap B_{k}, A_{3} \cap B_{k}\right)
$$


cioè

IV $\Phi\left(A_{1} ; A_{2}, A_{3}\right)=\sum_{k=1}^{3} \Phi\left(A_{\cap} B-\left(A_{2} \cup A_{3}\right) \cap B_{k} ; A_{2} \cap B_{k}, A_{3} \cap B_{k}\right)$

Si è così riconosciato che condizione necessaria perchè il guadagno di informazione $\Phi$ definisca una misura della quantità di informazione, $\theta$ che siano da esso verificate le relazioni I.-IV.

\section{7. - Caratterizzazione dell'informazione.}

Si può ora stabilire il seguente teorema di caratterizzazione della quan. tità di informazione $H$ mediante la funzione di evento $h(A)$ e il guadagno di informazione $\Phi(A ; B, C)$ :

Teorema 2. - Condizione necessaria e sufficiente perchè l'applicazione $\mathscr{E} \stackrel{H}{\rightarrow} \bar{R}^{+}$definila dalla 4.1 sia una misura della quantità di informazione, è che:

le funzioni $h(A)=H_{1}(A)$ e $\Phi(A ; B, C)$ verifichino le relazioni seguenti:

a)

$$
h(A) \in \bar{R}^{+}, \quad\{A\} \in \mathcal{G}
$$

$$
h(A \cap B)=h(A)+h(B) \quad\{A\} \in \mathfrak{d},\{B\} \in \mathfrak{d}
$$

I.

$$
\Phi(A ; B, C) \geq 0 \quad\{A, B, C\} \in \mathcal{E}
$$

II.

$$
\Phi(A ; Q, C)=0 \quad\{A, C\} \in \mathcal{E}
$$

III. $\Phi(C \cup D ; A, B)+\Phi(D ; A \cup B, C)=\Phi(B \cup D ; A, C)+\Phi(D ; A \cup C, B)$

$$
\{A, B, C, D\} \in \mathcal{E}
$$

IV. $\Phi\left(A_{1}, A_{2}, A_{3}\right)=\sum_{k=1}^{3} \Phi\left(A_{\cap} B-\left(A_{2} \cup A_{3}\right) \cap B_{k} ; \quad A_{2} \cap B_{k}, \quad A_{3} \cap B_{k}\right)$

$$
\left\{A_{1}, A_{2}, A_{3}\right\} \in \mathfrak{d}, \quad\left\{B_{1}, B_{2}, B_{3}\right\} \in \mathfrak{K}, \quad A=\bigcup_{k=1}^{3} A_{k}, \quad B=\bigcup_{k=1}^{\mathfrak{3}} B_{k}
$$

Dimostraziont. - Per come sono state dedotte le relazioni $a) \cdot b$ ), I.-IV., ò immediato riconoscere che esse sono necessarie perchè, ai sensi della (4.1), le funzioni $h(A)$ e $\Phi(A ; B, C)$ definiscano una misura di informazione.

IC poi immediato verificare che se $Z(\Pi)$ è una misura della quantità di informazione, ciò̀ se soddisfa agli assiomi i)-iii), e se $h(A)$ soddisfa le rela. zioni $a) \cdot b$ ), l'applicazione $\underset{\mathcal{O}}{\stackrel{H}{\longrightarrow}} \bar{R}^{+}$con $H(\Pi)=h(\underset{A \in \Pi}{\bigcup} A)+Z(\Pi)$ è una misura
della quantità di informazione. 
Basterà dunque verificare che se la funzione $\Phi(A ; B, C)$ soddisfa le proprietà I.-IV., la funzione $Z$ da essa definita soddisfa gli assiomi i)-iii).

È immediato riconoscere anzitutto, sulla base della proprietà 1. , ehe la funzione

$$
Z\left(A_{1}, A_{2}, \ldots, A_{n}\right)=\sum_{i=0}^{n-2} \Phi\left(\bigcup_{k=0}^{i} A_{k}, A_{i+1}, \bigcup_{n=i+2}^{i} A_{k}\right)
$$

è un'applicazione delle $n$-ple ordinate $A_{1}, \ldots, A_{n}$, tali che $\left\{A_{1}, \ldots, A_{n}\right\} \in \mathcal{B}$, in $\bar{R}^{+}$.

D'altra parte, per la (7.1), ò anche (per $1<j<n-1)$ :

$$
\begin{aligned}
& Z\left(A_{1}, \ldots, A_{j-1}, A_{j}, A_{j+1}, \ldots, A_{n}\right)-Z\left(A_{1}, \ldots, A_{j-1}, A_{j+1}, A_{i}, \ldots, A_{n}\right)= \\
& \quad=\Phi\left(A_{j} \cup\left[\bigcup_{k=0}^{j-1} A_{k}\right], A_{j+1},\left[\bigcup_{n=j+2}^{n} A_{n}\right]\right)+\Phi\left(\bigcup_{k=0}^{j-1} A_{k}, A_{j}, A_{i+1} \cup\left[\bigcup_{n=j+2}^{n} A_{h}\right]\right)- \\
& \quad-\Phi\left(A_{j+1} \cup\left[\bigcup_{k=0}^{j-1} A_{k}\right], A_{j}, \bigcup_{n=j+2}^{n} A_{h}\right)-\Phi\left(\bigcup_{k=0}^{j-1} A_{k}, A_{j+1}, A_{j} \cup\left[\bigcup_{n=j+2}^{n} A_{h}\right]\right)
\end{aligned}
$$

Posto in essa $A=\bigcup_{k=j+2}^{n} A_{k}, B=A_{j+1}, C=A_{j}, D=\bigcup_{h=0}^{j} A_{h}$ dalla III. e dal. la conseguente (6.7) si ha:

$$
\begin{aligned}
& \Phi\left(A_{j} \cup\left[\bigcup_{k=0}^{i-1} A_{k}\right], A_{i+1},\left[\bigcup_{n=j+2}^{n} A_{h}\right]\right)+\Phi\left(\bigcup_{k=0}^{j-i} A_{k}, A_{j}, A_{i+1} \cup\left[\bigcup_{n=i+2}^{n} A_{n}\right]\right)- \\
& -\Phi\left(A_{j+1} \cup\left[\bigcup_{k=0}^{j-2} A_{k}\right], A_{j}, \bigcup_{h=j+2}^{n} A_{h}\right)-\Phi\left(\bigcup_{k=0}^{j-1} A_{k}, A_{j+1}, A_{j} \cup\left[\bigcup_{h=j+2}^{n} A_{h}\right]\right)=0
\end{aligned}
$$

e quindi

$$
Z\left(A_{1}, \ldots, A_{j-1}, A_{j}, A_{j+1}, \ldots, A_{n}\right)=Z\left(A_{1}, \ldots, A_{j-1}, A_{j+1}, A_{j}, \ldots, A_{n}\right)
$$

Col medesimo procedimento si dimostra altresì che è

$$
Z\left(A_{1}, A_{2}, \ldots, A_{n-1}, A_{n}\right)=Z\left(A_{2}, A_{1}, \ldots, A_{n-1}, A_{n}\right)
$$

mentre dalla (7.1) e della (6.7) segue direttamente

$$
Z\left(A_{1}, \ldots, A_{n-1}, A_{.}\right)=Z\left(A_{1}, \ldots, A_{n}, A_{n-1}\right)
$$

Con eio si riconosce che la funzione $Z$, completata mediante l'identificazione $Z(\{A, Q\})=Z\left\{(A\}\right.$, è un'applicazione di $\mathcal{G}$ in $\bar{R}^{+}$, essendo totalmente simmetrica rispetto agli argomenti.

Siano ora

$$
\Pi_{1} \equiv\left\{A_{1}, \ldots, A_{n-2}, A_{n-1} \cup A_{n}\right\}, \quad \Pi_{2} \equiv\left\{A_{1}, \ldots, A_{n}\right\} \in \mathcal{G} .
$$


Sempre dalla espressione di $Z$ in termini della funzione $\Phi$ si ha

$$
Z\left(\Pi_{2}\right)-Z\left(\Pi_{1}\right)=\Phi\left(\bigcup_{k=0}^{n-2} A_{k}, A_{n-1}, A_{n}\right)
$$

e da questa per la proprietà I.:

$$
Z\left(\Pi_{1}\right) \leq Z\left(\Pi_{2}\right)
$$

La (7.3) consente, per indnzione, di stabilire che in generale si ha

$$
\Pi_{1}<\Pi_{2} \Rightarrow Z\left(\Pi_{1}\right) \leq Z\left(\Pi_{2}\right)
$$

pertanto l'assioma i) è verificato.

La (7.1) assicura altresì che l'applicazione $Z$ verifica la proprietà 4 ), e quindi anche - come de facile riconoscere - l'assioma iii).

Per completare la verifica dell'asserto, si deve ora dimostrare che (assioma iii)) se

$$
\begin{aligned}
& \Pi_{1} \equiv\left\{A_{1}, \ldots, A_{m}\right\} \in \mathfrak{d}, \quad \Pi_{2} \equiv\left\{B_{1}, \ldots, B_{n}\right\} \in \mathfrak{d} \mathfrak{\mathcal { L }}, \grave{\mathrm{e}} \\
& H\left(\Pi_{1} \Pi_{2}\right)=H\left(\Pi_{1}\right)+H\left(\Pi_{2}\right)
\end{aligned}
$$

Nel caso $m=n=1$, essendo $H(\{A\})=h(A)$, per la $a) \dot{~}$

$$
H(\{A\}(B\})=H(\{A\})+H(\{B\})
$$

e la (7.4) è dunque verificata. Se è $m=1, n \geq 2$, risulta

$$
\begin{aligned}
& H\left(\{A\} \Pi_{2}\right)=H(\{A\}\{B\})+Z\left(\{A\} \Pi_{2}\right)= \\
& \quad=H(\{A\})+H(\{B\})+Z\left(\{A\} \Pi_{2}\right), \quad \text { essendo } \quad B=\bigcup_{i=1}^{n} B_{i}
\end{aligned}
$$

D'altra parte ̀̀

$$
Z\left(\{A\} \Pi_{2}\right)=\sum_{i=0}^{n-2} \Phi\left(A \cap \bigcup_{k=0}^{i} B_{k}, A \cap B_{i+1}, A_{\cap} \bigcup_{h=i+2}^{i} B_{h}\right)
$$

Dalla IV., posto $B_{2}=B_{3}=Q, B_{1}=B$, si ha

$$
\Phi\left(A_{1} ; A_{z}, A_{3}\right)=\Phi\left(B \cap A_{1}, B \cap A_{2}, B \cap A_{3}\right)
$$

e ancora, posto

$$
A_{1}=\bigcup_{k=0}^{i} B_{k}, \quad A_{2}=B_{i+2}, \quad A_{3}=\bigcup_{h=i+2}^{n} B_{h}, \quad B=A,
$$




$$
\Phi\left(\bigcup_{k=0}^{i} B_{k}, B_{i+1}, \bigcup_{h=i+2}^{n} B_{h}\right)=\Phi\left(A \cap \bigcup_{k=0}^{i} B_{k}, A \cap B_{i+1}, A \cap \bigcup_{h=i+2}^{n} B h\right)
$$

Da quest'ultima relazione segue:

$$
Z\left(\{A\} \Pi_{2}\right)=Z\left(\Pi_{2}\right)
$$

che giustifica ulteriormente la denominazione di informazione condizionale attribuita alla applicazione $\mathcal{E}_{\stackrel{Z}{\rightarrow}}^{\rightarrow} \bar{R}^{+}$e prova infine che

$$
H\left(\{A\} \Pi_{2}\right)=H(\{A\})+H(\{B\})+Z\left(\Pi_{2}\right)=H(\{A\})+H\left(\Pi_{2}\right)
$$

Sia ora $m=2, n \geq 2$. Si ha successivamente

$$
\begin{array}{r}
H\left(\Pi_{1} \Pi_{2}\right)=H\left(\{A\} \Pi_{2}\right)+\sum_{k=1}^{n} \Phi\left(A \cap B-A_{\cap} B_{k}, A_{1} \cap B_{k}, A_{2} \cap B_{k}\right)= \\
=H(\{\boldsymbol{A}))+\sum_{k=1}^{n} \Phi\left(A_{\cap} B-A_{\cap} B_{k}, A_{1} \cap B_{k}, A_{2} \cap B_{k}\right)+H\left(\Pi_{2}\right)
\end{array}
$$

Per la II. e la IV., posto $B_{3}=Q$, si ha

$$
\Phi\left(A_{1}, A_{2}, A_{3}\right)=\sum_{k=1}^{2} \Phi\left(A_{\cap} B-\left(A_{2} \cup A_{3}\right) \cap B_{k}, A_{2} \cap B_{k}, A_{3} \cap B_{k}\right)
$$

Si può ora riconoscere che se è

$$
\begin{aligned}
& \Phi\left(A_{1}, A_{2}, A_{3}\right)=\sum_{k=1}^{n-2} \Phi\left(A \cap B-\left(A_{2} \cup A_{3}\right) \cap B_{k}, A_{2} \cap B_{k}, A_{3} \cap B_{k}\right)+ \\
& \quad+\Phi\left(A_{\cap} B-\left(A_{2} \cup A_{3}\right) \cap\left(B_{n-1} \cup B_{n}\right), A_{2} \cap\left(B_{n-1} \cup B_{n}\right), A_{3} \cap\left(B_{n-1} \cup B_{n}\right)\right)
\end{aligned}
$$

è anche

$$
\Phi\left(A_{1}, A_{2}, A_{3}\right)=\sum_{k=1}^{n} \Phi\left(A \cap B-\left(A_{2} \cup A_{3}\right) \cap B_{k}, A_{2} \cap B_{k}, A_{3} \cap B_{k}\right)
$$

Infatti dalla IV, sostituendo a $B_{\imath}, B_{2}, B_{3}$ rispettivamente $\bigcup_{k=1}^{n-2} B_{k}, B_{n-1}$, $B_{n}$, si ha

$$
\begin{aligned}
& \Phi\left(A_{1}, A_{2}, A_{3}\right)=\Phi\left(A_{\cap} B-\left(A_{2} \cup A_{3}\right) \cap \bigcup_{k=1}^{n-2} B_{k}, A_{2} \cap \bigcup_{k=1}^{n-2} B_{k}, A_{3} \cap \bigcup_{k=1}^{n-2} B_{k}\right)+ \\
& \quad+\Phi\left(A_{\cap} B-\left(A_{2} \cup A_{3}\right) \cap B_{n-1}, A_{2} \cap B_{n-1}, A_{8} \cap B_{n-1}\right)+ \\
& \quad+\Phi\left(A_{\cap} B-\left(A_{2} \cup A_{3}\right) \cap B_{n}, A_{2} \cap B_{n}, A_{3} \cap B_{n}\right)
\end{aligned}
$$


Analogamente sostituendo in IV. a $B_{1}, B_{2}, B_{3}$ rispettivamente $\bigcup_{k=1}^{n-2} B_{k}$, $B_{n-1} \cup B_{n}, Q$, si ha

$$
\begin{gathered}
\Phi\left(A_{1}, A_{2}, A_{3}\right)=\Phi\left(A \cap B-\left(A_{2} \cup A_{3}\right) \cap \bigcup_{k=1}^{n-2} B_{k}, A_{2} \cap \bigcup_{k=1}^{n-2} B_{k}, A_{3} \cap \bigcup_{k=1}^{n-2} B_{k}\right)+ \\
\quad+\Phi\left(A \cap B-\left(A_{2} \cup A_{3}\right) \cap\left(B_{n-1} \cup B_{n}\right), A_{2} \cap\left(B_{n-1} \cup B_{n}\right), A_{3} \cap\left(B_{n-1} \cup B_{n}\right)\right)
\end{gathered}
$$

Dal confronto della (7.13) con la (7.14) si ha infine:

$$
\begin{gathered}
\Phi\left(A_{\cap} B-\left(A_{2} \cup A_{3}\right) \cap\left(B_{n-1} \cup B_{n}\right), A_{2} \cap\left(B_{n-1} \cup B_{n}\right), A_{3} \cap\left(B_{n-1} \cup B_{n}\right)\right)= \\
\quad=\Phi\left(A_{\cap} B-\left(A_{2} \cup A_{3}\right) \cap B_{n-1}, A_{2} \cap B_{n-1}, A_{3} \cap B_{n-1}\right)+ \\
\quad+\Phi\left(A_{\cap} B-\left(A_{2} \cup A_{3}\right) \cap B_{n}, A_{2} \cap B_{n}, A_{3} \cap B_{n}\right)
\end{gathered}
$$

Con ciò la (7.12) è verificata per ogni valore dell'intero $n \geq 2$. Sosti. tuendo ora in esse $A_{1}, A_{2}, A_{3}$ rispettivamente con $\theta, A_{1}, A_{2}$ si ha

$$
\Phi\left(Q, A_{1}, A_{2}\right)=Z\left(\left\{A_{1}, A_{2}\right\}\right)=\sum_{k=1}^{n}\left(A_{\cap} B-A_{\cap} B_{k}, A_{1} \cap B_{k}, A_{2} \cap B_{k}\right)
$$

La (7.9) conduce così a

$$
\begin{aligned}
& H\left(\Pi_{1} \Pi_{2}\right)=H(\{A\})+Z\left(\left\{A_{1}, A_{2}\right)\right)+H\left(\Pi_{2}\right)= \\
& =H\left(\Pi_{1}\right)+H\left(\Pi_{2}\right)
\end{aligned}
$$

Quest'ultima relazione prova altresì che per $m=2, n \geq 2$, ̊̀

$$
Z\left(\Pi_{1} \Pi_{2}\right)=Z\left(\Pi_{1}\right)+Z\left(\Pi_{2}\right)
$$

Sia ora $m>2, n>2$; ̀̀

$$
H\left(\Pi_{1} \Pi_{2}\right)=H(\{A\})+H(\{B\})+Z\left(\Pi_{1} \Pi_{2}\right)
$$

Per provare la completa validità della (7.4) basterà dunque dimostrare che è

$$
Z\left(\Pi_{1} \Pi_{2}\right)=Z\left(\Pi_{1}\right)+Z\left(\Pi_{2}\right)
$$

Consideriamo dapprima il caso $m=3, n>2$. Si ha:

$$
Z\left(\Pi_{2} \Pi_{2}\right)=Z\left(\{A\}_{1}\right)+\sum_{k=1}^{n} \Phi\left(A \cap B-\left(A_{2} \cup A_{3}\right) \cup B_{k}, A_{2} \cap B_{k}, A_{3} \cap B_{k}+\right.
$$




$$
+\sum_{k=1}^{n} \Phi\left(A \cap B-A_{\cap} B_{k}, A_{1} \cap B_{k},\left(A_{2} \cup A_{3}\right) \cap B_{k}\right)
$$

e quindi per la (7.12)

$$
Z\left(\Pi_{1} \Pi_{2}\right)=Z\left(\{A\} \Pi_{2}\right)+\Phi\left(A_{1}, A_{2}, A_{3}\right)+\Phi\left(Q, A_{1}, A_{2} \cup A_{3}\right)
$$

Dalla (7.8) e dalla espressione di $Z\left(\Pi_{1}\right)$ si ha poi

$$
Z\left(\Pi_{1} \Pi_{2}\right)=Z\left(\Pi_{1}\right)+Z\left(\Pi_{2}\right)
$$

cioè l'asserto.

Sia infine $m>3, n>3$. Posto

$$
\Pi_{1}^{\prime} \equiv\left\{A_{1}, A_{2}, \ldots, A_{m-2}, A_{m-1} \cup A_{m}\right\}
$$

dalla iii) si ha:

$$
Z\left(\Pi_{1} \Pi_{2}\right)=Z\left(\Pi_{1}^{\prime} \Pi_{2}\right)+\sum_{k=1}^{n} \Phi\left(A_{\cap} B-\left(A_{m-1} \cup A_{m}\right) \cap B_{k}, A_{m-1} \cap B_{k}, A_{m} \cap B_{k}\right)
$$

e per la (7.12)

$$
Z\left(\Pi_{1} \Pi_{2}\right)=Z\left(\Pi_{1}^{\prime} \Pi_{2}\right)+\Phi\left(\bigcup_{i=1}^{m-2} A_{i}, A_{m-1}, A_{m}\right)
$$

pertanto, se la (7.17) è vera per i valori $m-1$ ed $n$ :

$$
Z\left(\Pi_{1} \Pi_{2}\right)=Z\left(\Pi_{1}^{\prime}\right)+\Phi\left(\bigcup_{i=1}^{n} A_{i}, A_{m-1}, A_{m}\right)+Z\left(\Pi_{2}\right)
$$

ma per la iii) ̀̀

$$
Z\left(\Pi_{1}\right)=Z\left(\Pi_{1}^{\prime}\right)+\Phi\left(\bigcup_{i=1}^{m-2} A_{i}, A_{m-1}, A_{m}\right)
$$

亡̀ dunque:

$$
Z\left(\Pi_{1} \Pi_{2}\right)=Z\left(\Pi_{1}\right)+Z\left(\Pi_{2}\right)
$$

Cio conclude la dimostrazione dell'asserto.

\section{8. - L'informazione di Shannon-Rényi.}

Si possono ora stabilire alcune condizioni necessarie e sufficienti perohè la misura dell'informazione $H$ di un'esperienza incompleta sia data dalla formula di Shannon-Rényi.

A questo proposito si hanno i seguenti teoremi 
TEOREMa 3. - Se la funzione $h$ è un'informazione di evento $J(A)$, munita di una legge di composizione $J(A \cup B)=J(A) T J(B)(A \cap B=Q)[1]$ e se $\Phi(A ; B, C)$ è una funzione continua dei valori di J sugli insiemi $A \cup B \cup C, B, C: \Phi(A ; B, C)=$ $=\varphi(J(A \cup B \cup C), J(B), J(C))$, allora $H_{n}\left(A_{1}, \ldots, A_{n}\right)$ è una funzione continua dei valori di $J$ sugli insiemi $A_{1}, \ldots A_{n}$.

Dimostrazione. - Basta osservare che per la (4.1) ̀

$$
\begin{gathered}
H_{n}\left(A_{1}, \ldots, A_{n}\right)=J\left(\bigcup_{i=1}^{n} A_{i}\right)+\sum_{i=0}^{n-2} \Phi\left(\bigcup_{k=0}^{i} A_{k}, A_{i}, \cup A_{k}\right)= \\
=\prod_{i=1}^{n} J\left(A_{i}\right)+\sum_{i=0}^{n-2} \varphi\left(\prod_{k=0}^{i} J\left(A_{k}\right), J\left(A_{i}\right), \prod_{k=i+2}^{n} J\left(A_{k}\right)\right)
\end{gathered}
$$

e ricordare la proprietà di continuità della legge di composizione $T$.

Se poi $J$ è un'informazione shannoniana (delle condizioni sufficienti perchè ciò accada sono state date da J. Kampé de Fériet e B. Forte: Information et probabilité, ...), ciò̀ se

$$
J(A \cup B)=J(A) T J(B)=-\log \left[e^{-J(A)}+e^{-J(B)}\right],
$$

si ha in più il

TeORema 4. - Condizione necessaria e sufficiente perchè l'informazione $H_{n}\left(A_{1}, \ldots, A_{n}\right)$ sia un'informazione di Shannon-Rényi:

è che:

$$
H_{n}\left(A_{1}, \ldots, A_{n}\right)=\frac{\sum_{k=1}^{n} J\left(A_{k}\right) e^{-J\left(A_{k}\right)}}{\sum_{k=1}^{n} e^{-J\left(A_{k}\right)}}
$$

\section{1) J sia di tipo shannoniano}

2) siano verificate le ipotesi del teorema 3.

Dimostrazione. - Dimostriamo anzitutto che la condizione è sufficiente. Introduciamo la funzione $\biguplus$ definita da

$$
\Psi\left(e^{-x} ; e^{-y}, e^{-z}\right)=\varphi(x ; y, z
$$

La proprieta I. per il guadagno di informazione implica

$$
\begin{aligned}
& \psi(u ; v, w)=\psi(u ; w, v) \geq 0 \\
& (v+w \leq u ; 0 \leq v \leq 1 ; 0 \leq w \leq 1 ; 0 \leq u \leq 1)
\end{aligned}
$$


La proprietà II. tenendo conto $\operatorname{ch} \theta$ è $J(\theta)=+\infty$, dà

$$
\psi(u ; 0, v)=0 \quad(0 \leq v \leq u \leq 1)
$$

La proprietà III. implica

$$
\begin{gathered}
\psi\left(v ; u_{1}, u_{2}\right)+\psi\left(v ; u_{1}+u_{2}, u_{3}\right)=\psi\left(v ; u_{1}, u_{3}\right)+\psi\left(v ; u_{1}+u_{3}, u_{2}\right) \\
\left(u_{1}+u_{2}+u_{3} \leq v \leq 1 ; u_{1} \geq 0, u_{2} \geq 0, u_{3} \geq 0\right) .
\end{gathered}
$$

La proprietà IV. implica infine

$$
\begin{gathered}
\Psi\left(u_{1}+u_{2}+u_{3}, u_{2}, u_{3}\right)=\sum_{=1}^{s} \psi\left(\Sigma k_{i} \Sigma v_{k}, u_{2} v_{k}, u_{3} v_{k}\right) \\
\left(u_{i} \geq 0, v_{i} \geq 0 \text { per } i=1,2,3 ; u_{1}+u_{2}+u_{3} \leq 1 ; v_{1}+v_{2}+v_{3} \leq 1\right)
\end{gathered}
$$

Da quest'equazione segue che la funzione $\psi(u, v, w)$ è omogenea di grado zero; infatti, posto $v_{1}=t, v_{2}=v_{3}=0$, si ha

$$
\psi\left(u_{1}+u_{2}+u_{3}, u_{2}, u_{3}\right)=\psi\left(u_{1} t+u_{2} t+u_{3} t, u_{2} t, u_{3} t\right)
$$

Posto allora

$$
F(u, v, w)=u \Psi(u ; v, w)
$$

la funzione $F$ risulta omogenea di primo grado nelle sue variabili, e può quindi essere prolungata per omogeneità in modo da risultare definita per $u \geq 0$, $v \geq 0, w \geq 0, v+w \leq u$.

In più, dalla relazione (che segue da (8.4))

$$
\psi\left(u_{1}+u_{2}+u_{3}, u_{2}, u_{3}\right)=\sum_{i=1}^{m} \psi\left(\Sigma u \Sigma v, u_{2} v_{k}, u_{3} v_{k}\right)
$$

posto $v_{k}=1 / m, k=1, \ldots, m$, si ha

$$
F(u, v, w)=F(m u, v, w)
$$

D'altra parte, se $u^{\prime}$ e $u^{\prime \prime}$ sono numeri reali nell'intervallo $(0,1)$ tali che $u^{\prime}=\frac{r}{s} u^{\prime \prime}(r, s$ interi $)$, si ha

$$
\begin{gathered}
F\left(u^{\prime}, v, w\right)-F\left(u^{\prime \prime}, v, w\right)=F\left(\frac{r}{s} u^{\prime \prime}, v, w\right)-F\left(u^{\prime \prime}, v, w\right)= \\
\quad=\frac{1}{s} F\left(r u^{\prime \prime}, s v, s w\right)-F\left(u^{\prime \prime}, v, w\right)=\frac{1}{s} F\left(u^{\prime \prime}, s v, s w\right)- \\
\quad-F\left(u^{\prime \prime}, v, w\right)=F\left(\frac{u^{\prime \prime}}{s}, v, w\right)-F\left(u^{\prime \prime}, v, w\right)=0
\end{gathered}
$$

Per la continuità della fanzione $\Phi$, che implica quella della funzione $F$, si ha che per ogni coppia di numeri reali $u^{\prime}, u^{\prime \prime}$ in $(0,1)$ è $F\left(u^{\prime}, v, w\right)=F\left(u^{\prime \prime}\right.$, $v, w)$, ciò̀ la funzione $F(u, v, w)$ non dipende dalla variabile $u$. 
Si può pertanto porre

$$
F(u, v, w)=f(v, w)
$$

Le proprietà $(8.1)-(8.3)$ in termini della funzione $f(v, w)$ divengono allora

$$
f(x, y)=f(y, x) \geq 0 \quad \text { per } \quad x, y \in(0,1)
$$

$$
f(0, y)=0, \quad y \in(0,1)
$$

$$
f(x, y)+f(x+y, z)=f(x, z)+f(x+z, y)
$$

In più, la funzione $f(x, y)$ è una funzione omogenea dí grado uno nelle sue variabili. Utilizzando questa proprietà, insieme alla simmetria della $f$, la (8.3) può seriversi nella forma

$$
f(y, x)-f(z, x)=f(x+z, y)-f(x+y, z)
$$

e posto

$$
g\left(\frac{x}{y}\right)=f\left(\frac{x}{y}, 1\right)
$$

si ha

$$
x g\left(\frac{y}{x}\right)-x g\left(\frac{z}{x}\right)=y g\left(\frac{x+z}{y}\right)-z g\left(\frac{x+y}{z}\right)
$$

Posto infine $y / x=u, z / x=v$, si ha

$$
g(u)-g(v)=u g\left(\frac{1+v}{u}\right)-v g\left(\frac{1+u}{v}\right)
$$

A quest'equazione funzionale nell'incognita funzione $g(x)$ va aggiunta l'equazione che si ottiene dalla simmetria e dalla omogeneita della funzione $f$ :

$$
x f\left(\frac{y}{x}, 1\right)=y f\left(\frac{x}{y}, 1\right)
$$

$\operatorname{ciò̀}$

$$
g(u)=u g\left(\frac{1}{u}\right)
$$

dovendo poi essere

$$
g(0)=0, \quad g(u) \geq 0 .
$$


Si può ora dimostrare che il sistema di equazioni funzionali

$$
\left\{\begin{array}{l}
g(u)-g(v)=u g\left(\frac{1+v}{u}\right)-v g\left(\frac{1+u}{v}\right) \\
g(u)=u g\left(\frac{1}{u}\right)
\end{array}\right.
$$

con le condizioni (8.7) ammette quale unica soluzione continua la funzione

$$
g(u)=p\{(u+1) \log (u+1)-u \log u\}
$$

Dalla (8.5) si ha, integrando nella variabile $v$ :

$$
v g(u)=\int_{0}^{v} g(\xi) d \xi+u \int_{0}^{v} g\left(\frac{\xi+1}{u}\right) d \xi-\int_{0}^{v} \xi g\left(\frac{u+1}{\xi}\right) d \xi
$$

e usufruendo della $(8.6)$

$$
v g(u)=\int_{0}^{v} g(\xi) d \xi+u^{2} \int_{1 / u}^{\frac{v+1}{u}} g(\xi) d \xi-(u+1)^{2} \int_{0}^{\frac{v}{u+1}} g(\xi) d \xi
$$

il che dimostra che $g(u) \in C^{\mathrm{1}}(0,+\infty)$.

Derivando quindi primo e secondo membro di questa equazione rispetto alla variabile $u$, si ha

$$
\begin{aligned}
& v g^{\prime}(u)=2 u \int_{\frac{1}{u}}^{\frac{v+1}{u}} g(\xi) d \xi \quad(v+1) g\left(\frac{v+1}{u}\right)+g\left(\frac{1}{u}\right)- \\
& -2(u+1) \int_{0}^{\frac{u+1}{v}} g(\xi) d \xi+v g\left(\frac{v}{u+1}\right)
\end{aligned}
$$

e questa relazione dimostra ehe $g^{\prime}(u) \in C^{2}(0, \infty)$.

Derivando allora primo e secondo membro della (8.5) rispetto alla $u$, si ha

$$
g^{\prime}(u)=g\left(\frac{v+1}{u}\right) \quad \frac{v+1}{u} g^{\prime}\left(\frac{v+1}{u}\right)-g^{\prime}\left(\frac{u+1}{v}\right)
$$

e scambiando $u$ con $v$ :

$$
g^{\prime}(v)=g\left(\frac{u+1}{v}\right)-\frac{u+1}{v} g^{\prime}\left(\frac{u+1}{v}\right)-g^{\prime}\left(\frac{v+1}{u}\right)
$$


e derivando ulteriormente rispetto a $u$ :

$$
0=-\frac{u+1}{v^{2}} g^{\prime \prime}\left(\frac{u+1}{v}\right)+\frac{v+1}{u^{2}} g^{\prime \prime}\left(\frac{v+1}{u}\right)
$$

Ponendo quindi $v=u+1$

$$
\frac{1}{u+1} g^{\prime \prime}(1)=\frac{u+2}{u^{2}} g^{\prime \prime}\left(\frac{u+2}{u}\right)
$$

Posto infine $\frac{u+2}{u}=x, g^{\prime \prime}(1)=k$, si ha

$$
g^{\prime \prime}(x)=-2 k\left[\frac{1}{x+1}-\frac{1}{x}\right]
$$

che implica

$$
g(x)=-2 k[(x+1) \log (x+1)-x \log x]+c_{1} x+c_{2}
$$

Ma essendo $\lim g(x)=0$, dovrà essere $c_{2}=0$.

D'altra parte, la equazione (8.5) implica

$$
c_{1} u-c_{2} v=c_{1} v-c_{2} u
$$

da cui $c_{1}=0$. Posto infine $-2 k=p$, si perviene a

$$
g(x)=p[(x+1) \log (x+1)-x \log x]
$$

É immediato riconoscere a questo punto che alla funzione (8.8) corrisponde la funzione

$$
\psi(u, v, w)=\frac{p}{u}[(v+w) \log (v+w)-v \log v-w \log w]
$$

cui corrispoude l'informazione

$$
H_{n}\left(A_{1}, \ldots, A_{n}\right)=\frac{\sum_{k=1}^{\imath} J\left(A_{k}\right) e^{-J\left(A_{k}\right)}}{\sum_{k=1}^{n} e^{-J\left(A_{k}\right)}}
$$

vale a dire la formula di Shannon-Rényi.

È immediato rieonoscere viceversa che la formula di Shannon-Rényi soddisfa tatte le condizioni del teorema 4.

\section{9. - Informazione di un'esperienza completa.}

Sia $\mathfrak{f}^{*}$ il sottoinsieme di $\mathfrak{b}$ formato dalle esperienze complete. La restrizione $H^{*}$ dell'applicazione $H$ ad $\mathscr{b}^{*}$ definisce un guadagno di informazione 
B. Forte - N. Pintacuda: Sull'informazione associata alle esperienze, ecc.

$\Phi^{*}$ che è la restrizione di $\Phi$ alle terne di eventi $A, B, C$ tali che $A \cup B \cup C=\Omega$.

Il guadagno di informazione $\Phi^{*}$ è dnnque in questo caso una applicazione $\Psi(A, B)=\Phi^{*}(\Omega-(A \cup B), A, B)$ delle coppie di eventi disgiunti $A, B$ in $\bar{R}^{+}$

Nel caso in cui $\Psi$ dipenda da $A$ e da $B$ tramite $i$ valori che la funzione $h$ assume in corrispondenza ad $A$ e a $B$, si ha

$$
\Psi(A, B)=\psi(h(A), h(B))
$$

Si supponga ora che la funzione $h$ sia un'informazione di evento $J(A)$ munita di una legge di composizione

$$
J(A \cup B)=J(A) \top J(B) \quad(A \cap B=Q)
$$

Detto

$$
\begin{aligned}
& \Gamma=\{(x, y): x=J(A), y=J(B), A \cap B=Q\} \\
& \Gamma_{i}=\{(x, y): x=J(A), y=J(B),\{A\} \in \mathcal{J},\{B\} \in \mathcal{J}\}
\end{aligned}
$$

e posto $G(x, y)=\psi(x, y)$, e ricordando che che $J(\Theta)=+\infty$, le condizioni 1)-4) si tradueono in

$$
G(x, y) \geq 0 \quad(x, y) \in \mathrm{I}^{\prime}
$$

$$
G(+\infty, y)=0
$$

$$
\begin{aligned}
& G(x, y)+G(x \top y, z)=G(x, z)+G(x \top z, y) \\
& (x, y) \in \Gamma, \quad(x, z) \in \Gamma, \quad(x \top y, z) \in \Gamma, \quad(x \top z, y) \in \Gamma
\end{aligned}
$$

$G\left(x_{2}, x_{3}\right)=G\left(x_{2}+y_{1}, x_{3}+y_{1}\right)+G\left(x_{2}+y_{2}, x_{3}+y_{2}\right)+$

$$
+G\left(x_{2}+y_{3}, x_{3}+y_{3}\right) \quad\left(y_{1} \top y_{2} \top y_{3}=0\right)
$$

Si puó facilmente dimostrare che queste proprietà sono equivalenti alle seguenti :

$$
\begin{gathered}
G(x, y) \geq 0 \quad(x, y) \in \Gamma \\
G(x, y)=G(y, x) \quad(x, y) \in \Gamma \\
G(x, y)+G(x \top y, z)=G(x, z)+G(x \top z, y)
\end{gathered}
$$$$
(x, y) \in \Gamma, \quad(x, z) \in \Gamma, \quad(x \mathrm{~T} y, z) \in \Gamma, \quad(x \mathrm{~T} z, y) \in \Gamma
$$

$$
\begin{aligned}
& G\left(x^{*}+x \top y, y^{*}+x \top y\right)=G\left(x^{*}+x, y^{*}+x\right)+G\left(x^{*}+y, y^{*}+y\right) \\
& \left(x, x^{*}\right) \in \Gamma_{i}, \quad\left(x, y^{*}\right) \in \Gamma_{i}, \quad\left(y, x^{*}\right) \in \Gamma_{i}, \quad\left(y, y^{*}\right) \in \Gamma_{i}
\end{aligned}
$$


Queste proprietà coincidono con le condizioni necessarie e sufficienti perchè la funzione $G$ definisca l'informazione di una esperienza completa senza probabilita, come trovasi dimostrato in B. FonTE: On the amount of information given by an experiment (in corso di stampa) [5].

\section{0. - Conelusioni.}

Gli assiomi ohe definiscono l'informazione di una esperienza incompleta ai sensi di quanto visto nel paragr. 7 danno lnogo ad una deduzione di tipo nuovo dell'informazione di Shannon-Rényi, e sono del tutto compatibili con gli assiomi che definiscono l'informazione di un'esperienza completa. Tattavia si riconosce che essi non implicano per ciò che riguarda le esperienze costituite da un solo evento le proprietà dell'informazione secondo Kampé de Fériet-Forte.

Pertanto l'informazione risulta definita per ciò che riguarda le esperienze incomplete dagli assiomi proposti nel paragrafo 2, per ciò che riguarda i singoli eventi dagli assiomi proposti da J. Kampé de Fériet e B. Forte. Cio d'altra parte rispecchia $i$ due punti di vista classicamente distinti illustrati nell'introduzione.

\section{BIBLIOGRAFIA}

[1] C. PrCard, Theorie des questionnaires, Paris, Gauthier-Villars, 1964.

[2] J. Kampé de Fŕriet, B. Forte, Information et probabilité, Comptes Rendus, 265, série A, 1967, p. 110, 142 e $\$ 500$.

[3] A. RÉxy, On measures of entropy and information, Proe. 4 th Berkeley Symposium on Math. Stat. Prob. 1960, vol, I, Univers. of California Press, Berkeley-Los Angeles 1961, p. 547-561.

[4] D. A. Kappos, Strukturtheorie der Wahrscheinlichkeitsfelder u.-räume, Berlin, Springer Verlag, 1960, p. 71 .

[5] B. ForTE, On the amount of information given by an experiment, in corso di stampa. 